\title{
Um Middleware para Gerenciamento de Sensores de Saúde Corporais em Dispositivos Móveis
}

\author{
Samuel Francisco Ferrigo ${ }^{1}$, Cristiano André da $\operatorname{Costa}^{1}$, Rodrigo da Rosa Righi $^{1}$ \\ ${ }^{1}$ Programa de Pós-graduação em Computação Aplicada (PIPCA) \\ Universidade do Vale do Rio dos Sinos (UNISINOS) \\ São Leopoldo - RS - Brasil \\ samuelffeedu.unisinos.br, \{cac,rrrighi\}@unisinos.br
}

\begin{abstract}
The use of IoT sensors to monitoring vital signs presents a series of challenges, such as the search for energy efficiency and the synchronization between sensors. A solution to these problems is presented by the MOMICARE model, a middleware for smartphones to manage a body wireless sensor network with emphasis on power consumption and synchronization of data sent by these sensors. As a scientific contribution, MOMICARE offers low power consumption and timing between the data collected by the sensors. The evaluation of the model was performed through simulations carried out in the Cooja software, presenting a reduction in energy consumption.
\end{abstract}

Resumo. O uso de sensores IoT para monitoramento de sinais vitais apresenta uma série de desafios, como a busca pela eficiência energética e a sincronização entre sensores. Uma solução para estes problemas é apresentada pelo modelo MOMICARE, um middleware para smartphones para gerenciamento de uma rede de sensores sem fio corporais com ênfase no consumo de energia e na sincronização dos dados enviados por esses sensores. Como contribuição científica, o MOMICARE oferece baixo consumo de energia e sincronismo entre os dados coletados pelos sensores. A avaliação do modelo foi realizada por meio de simulações efetuadas no software Cooja, apresentando redução no consumo de energia.

\section{Introdução}

A área da saúde, historicamente, consome muitos recursos financeiros. No Brasil, somente o governo federal investe quase $2 \%$ do PIB em saúde [IBGE 2015]. Ainda assim, em virtude de suas dimensões continentais, os serviços médicos do país são ineficientes e acessíveis a apenas uma parcela da população, principalmente àquelas que vivem nos grandes centros urbanos.

Nesse contexto, a mobile healthcare, também conhecida por $m$-health, torna-se uma aliada na melhora da eficiência em cuidados médicos [Merrell and Doarn 2014]. Nela, a Internet das Coisas, ou Internet of Things (IoT) [Riazul Islam et al. 2015], possui um papel fundamental. Isso permite, entre outras coisas, que problemas de saúde possam ser identificados antecipadamente e mais facilmente tratados, através de um monitoramento contínuo. Esse monitoramento consiste em medir a condição de saúde de um paciente, obtida através da medição de seus sinais vitais [Riazul Islam et al. 2015]. 
A $m$-health faz uso de sensores $I o T$ afixados ao corpo para a medição dos sinais vitais formando, dessa maneira, uma rede chamada de Wireless Body Area Network $(W B A N)$ [Castillejo et al. 2013]. Todavia, esses sensores dispõem de capacidade limitada de processamento e possuem um rádio integrado para transmissão dos dados coletados [Wang et al. 2015], sendo necessário um equipamento externo que receba e envie esses dados às aplicações externas que os requisitem, ou ainda que cada aplicação conecte-se diretamente ao(s) sensor(es) corporais desejados.

Nessa conjuntura, os smartphones podem atuar como um intermediário que receba esses dados e os enviem para as aplicações no momento em que haja uma rede disponível, conforme demonstrado na Figura 1. Como esse dispositivo está normalmente próximo de um corpo de uma pessoa, ele não teria problemas de comunicação pela distância. Ainda, em função de sua maior capacidade de processamento e armazenamento, o smartphone pode armazenar e transmitir os dados para aplicações médicas externas que os requisitem. Além disso, os smartphones possuem sensores internos - tais como acelerômetro e GPS que permitem que os dados capturados pelos sensores IoT possam ser complementados.

Entretanto, apenas o uso do smartphone não é suficiente para resolver todos os problemas relacionados ao uso de sensores de saúde corporais. Alguns desses desafios estão relacionados ao sincronismo entre os sensores e à duração da bateria desses dispositivos. O sincronismo é necessário para que o profissional médico possa realizar o diagnóstico correto, enquanto que a duração bateria de um sensor deve ser prolongada de forma a evitar que um sensor deixe de funcionar por falta de carga.

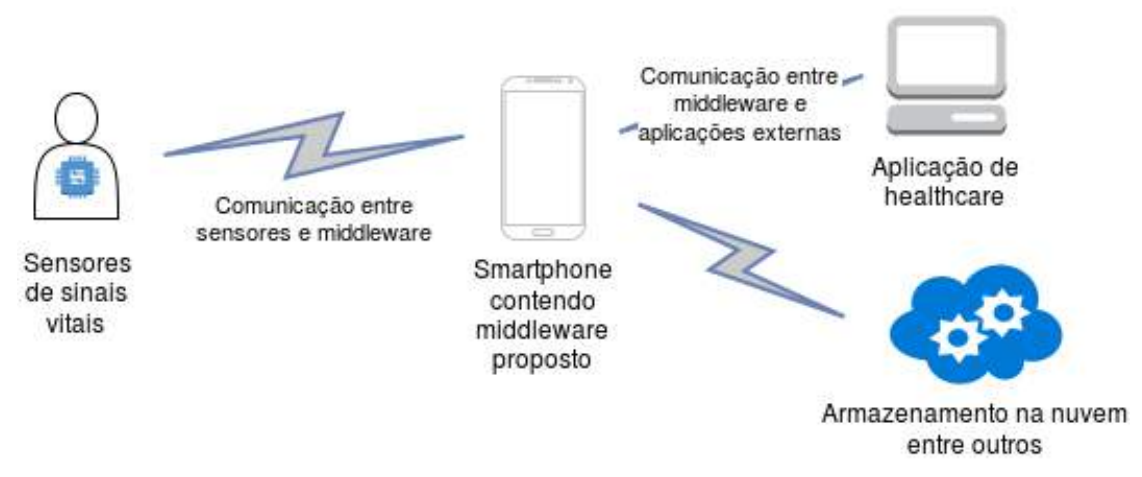

Figura 1. A proposta de solução para o problema.

Dessa forma, a principal contribuição científica desse trabalho é o desenvolvimento de um middleware específico que permita que os smartphones possam ser capazes de realizar o gerenciamento de sensores de saúde corporais, atacando os problemas de sincronização e consumo de energia que envolvem esses dispositivos. Essas são duas das lacunas que atualmente encontram-se abertas em trabalhos de pesquisa relacionados [Maia et al. 2015].

Este trabalho está dividido em seis seções. A Seção 2 apresenta os conceitos relacionados aos sensores IoT e a sua comunicação. A Seção 3 apresenta os trabalhos relacionados. A Seção 4 apresenta uma visão geral do modelo MOMICARE. A Seção 5 apresenta os resultados obtidos através de simulação. Por fim, a Seção 6 apresenta as conclusões do trabalho. 


\section{Redes WBAN}

Uma WBAN (Wireless Body Area Network) é uma rede de sensores sem fio (RSSF) utilizada para interconectar sensores implantados dentro de um corpo de uma pessoa ou dispostos sobre o corpo de uma pessoa [IEEE 2011], utilizados, entre outras aplicações, para cuidados médicos

Os sensores que formam uma rede WBAN, também conhecidos como motes, possuem baixa capacidade de armazenamento e processamento e possuem restrições de consumo de energia, visto que seu tamanho reduzido não permite que sejam utilizadas baterias com grande capacidade de armazenamento de energia [Goswami et al. 2016]. Os sensores são classificados em dois tipos: intrusivos e nãointrusivos [Shankar and Tomar 2016]. Os sensores intrusivos são inseridos dentro do corpo humano através de procedimento cirúrgico e são usados para medições específicas, como a verificação da pressão intra-craniana. Já os sensores não-intrusivos, também conhecidos como wearables, são fixados externamente ao corpo humano através de pingentes, braceletes, meias, entre outros, e normalmente realizam a medição de sinais vitais, como pressão arterial, temperatura corporal, batimento cardíaco, etc. Estes sensores normalmente estão em volta das mãos, em volta dos braços, em frente ou atrás do peito, ao redor da coxa, em volta do tornozelo ou próximo às orelhas.

Os sensores comunicam-se normalmente através dos padrões Bluetooth Low Energy (BLE) [SIG 2016] e IEEE 802.15.4 [IEEE 2011], de forma que a troca de dados entre esses dispositivos consuma o mínimo de energia. Dessa forma, esses dispositivos têm capacidade suficiente apenas para processar e repassar informação para uma estação-base para posterior diagnose e análise por outros dispositivos computacionais [Goswami et al. 2016].

Para poupar energia na comunicação de dados entre os dispositivos, os padrões $B L E$ e IEEE 802.15.4 utilizam basicamente as mesmas técnicas: longos períodos de inatividade na comunicação - também chamado de baixo ciclo de trabalho - e quadros com payload de poucos bytes. Esse último aspecto torna ambos padrões incompatíveis com o protocolo $I P$, visto que o cabeçalho deste é muito grande e complexo. Como consequência, sobrecargas na rede ocorrem frequentemente. Para contornar o problema, surgiu o padrão 6LowPAN [Riazul Islam et al. 2015]. Localizado entre a camada de rede e a camada de enlace do modelo $O S I$, tem a função de reduzir o cabeçalho do IPv6 para um formato que possa ser comportado nos quadros dos padrões $B L E$ e 802.15.4.

\section{Trabalhos Relacionados}

Para a concepção do modelo, foram considerados sete trabalhos que (1) permitiam a utilização de um smartphone com utilitário de verificação de saúde; (2) utilizavam algum tipo de rede de transmissão para coleta dos dados de sensores de uma RSSF e/ou que transmitiam dados coletados do próprio equipamento para uma aplicação externa e; (3) que possuíam algum tipo de integração com aplicações externas. O trabalho de [Lee et al. 2012] apresenta um sistema de monitoramento de saúde que funciona a partir de um smartphone. Através de um aplicativo específico, o smartphone armazena os dados recebidos dos sensores via Bluetooth e permite que um usuário visualize, em tempo real, os resultados. A proposta apresentada por [Lane et al. 2014] tem por objetivo monitorar o bem-estar de uma pessoa, por meio de um aplicativo para smartphone que monitora as 
atividades de uma pessoa por meio do uso dos sensores disponíveis nestes dispositivos. A partir dos dados coletados, o aplicativo realiza inferências sobre esses dados e transmite os resultados para procesamento na nuvem.

A solução de [Seeger et al. 2015] consiste num middleware voltado a eventos relacionados a um interesse específico, como exercícios físicos, telemedicina ou cuidados a idosos. As aplicações healthcare podem se inscrever em um ou mais interesses, por meio do método publish-subscribe, recebendo assim apenas os eventos desejados. O artigo de [Maia et al. 2015] propõe uma plataforma de middleware Web que interliga diversos sensores heterogêneos e notifica as condições de saúde de um paciente - bem como seus sinais vitais - a aplicações web por meio do padrão REST e no protocolo HTTP. O trabalho de [Jung et al. 2013] propõe um gateway doméstico voltado a cuidados médicos propondo dietas, exercícios e outros cuidados médicos ao usuário. Essas propostas são elaboradas a partir de decisões tomadas por um ambiente ubíquo e também permite que um profissional médico contate remotamente esse paciente através da solução.

A proposta de [Moser and Melliar-Smith 2015] aborda o uso de um smartphone voltado exclusivamente para o monitoramento de saúde de uma pessoa. Além de coletar dados de sensores de sinais vitais pré-determinados de uma WBAN, ele fornece diversas análises estatísticas sobre os dados coletados e também faz a correlação entre a atividade física e as medições fisiológicas. Por fim, [Castillejo et al. 2013] traz uma solução de $e$ health voltada para ambientes internos que interliga dispositivos IoT de healthcare que tem por objetivo monitorar a condição de saúde de um paciente quanto este está praticando algum exercício físico.

A Tabela 1 apresenta um comparativo entre os trabalhos relacionados. Como pontos relevantes, verifica-se a pouca preocupação quanto à segurança na transmissão de dados entre dispositivos de uma rede $W B A N$, a baixa escalabilidade da rede $W B A N$, a pouca eficiência energética na comunicação entre os dispositivos da $W B A N$, e a sincronização entre sensores de uma rede WBAN. O objetivo do modelo proposto neste trabalho é preencher as lacunas de sincronismo e eficiência energética dos sensores, de forma a permitir que os dados coletados por esses dispositivos possam ser sincronizados e transmitidos de maneira energeticamente eficiente.

\section{O Modelo MOMICARE}

O MOMICARE, acrônimo de MObile MIddleware for HealthCARE, é um modelo de middleware projetado para funcionar em smartphones para gerenciamento de sensores de saúde de uma WBAN. O middleware recebe os dados de diversos sensores de saúde, sincroniza-os e armazena-os internamente e, quando solicitado, retransmite-os às aplicações médicas que necessitem utilizá-los. Ele também centraliza o recebimento dos dados enviados pelos diversos sensores. Isso evita retransmissões desnecessárias e, consequentemente, poupa energia desses dispositivos. A sincronização dos dados recebidos é efetuada pelo próprio middleware conforme os dados enviados pelos sensores são recebidos. Dessa forma, o MOMICARE provê sincronismo aos dados enviados pelos sensores, bem como oferece uma maior eficiência energética a esses dispositivos.

A arquitetura do middleware é formada por quatro módulos: módulo de comunicação, módulo do dispositivo, módulo de integração e módulo de gerenciamento. Conforme a Figura 2, os sensores que formam a rede WBAN comunicam-se com o mid- 
Tabela 1. Comparativo entre os trabalhos relacionados

\begin{tabular}{|c|c|c|c|c|c|c|c|}
\hline $\begin{array}{l}\text { Critério/ } \\
\text { Trabalho }\end{array}$ & $\begin{array}{l}\text { Lee et al. } \\
2012\end{array}$ & $\begin{array}{l}\text { Lane et } \\
\text { al. } 2014\end{array}$ & $\begin{array}{l}\text { Seeger et } \\
\text { al. } 2015\end{array}$ & $\begin{array}{l}\text { Maia et } \\
\text { al. } 2015\end{array}$ & $\begin{array}{l}\text { Jung et } \\
\text { al. } 2013\end{array}$ & $\begin{array}{l}\text { Moser } \\
\text { and } \\
\text { Melliar- } \\
\text { Smith } \\
2015\end{array}$ & \begin{tabular}{ll}
\multicolumn{2}{c}{ Castillejo } \\
et & al. \\
2013 &
\end{tabular} \\
\hline $\begin{array}{l}\text { Segurança na } \\
\text { transmissão }\end{array}$ & Não & Não & Não & Não & Não & $\begin{array}{l}\text { Somente } \\
\text { Bluetooth }\end{array}$ & Não \\
\hline Escalabilidade & Não & Não & Não & Não & Não & Não & Sim \\
\hline $\begin{array}{l}\text { Interopera- } \\
\text { bilidade }\end{array}$ & $\begin{array}{l}\text { 3G, WiFi, } \\
\text { Bluetooth }\end{array}$ & 3G, WiFi & $\begin{array}{l}\text { WiFi, } \\
\text { Bluetooth }\end{array}$ & $\begin{array}{l}802.15 .4, \\
\text { Bluetooth }\end{array}$ & $\begin{array}{l}\text { WiFi, } \\
\text { Bluetooth }\end{array}$ & $\begin{array}{l}\text { 3G, WiFi, } \\
\text { Bluetooth }\end{array}$ & $\begin{array}{l}\text { 802.15.4, } \\
\text { WiFi, } \\
\text { Bluetooth }\end{array}$ \\
\hline $\begin{array}{l}\text { Eficiência } \\
\text { energética }\end{array}$ & Não & Sim & Não & Não & Não & Não & Não \\
\hline $\begin{array}{l}\text { Uso de } \\
\text { sensores } \\
\text { do próprio } \\
\text { smartphone }\end{array}$ & Não & $\begin{array}{l}\text { Acelerô- } \\
\text { metro, } \\
\text { micro- } \\
\text { fone }\end{array}$ & $\begin{array}{l}\text { Acelerô- } \\
\text { metro }\end{array}$ & Não & Não & $\begin{array}{l}\text { Acelerô- } \\
\text { metro, } \\
\text { micro- } \\
\text { fone }\end{array}$ & Não \\
\hline $\begin{array}{ll}\text { Captura } & \text { dos } \\
\text { dados } & \text { dos } \\
\text { sensores } & \\
\end{array}$ & $\begin{array}{l}\text { Em } \\
\text { qualquer } \\
\text { lugar }\end{array}$ & $\begin{array}{l}\text { Em qual- } \\
\text { quer lu- } \\
\text { gar }\end{array}$ & $\begin{array}{l}\text { Em } \\
\text { qualquer } \\
\text { lugar }\end{array}$ & $\begin{array}{l}\text { Locais } \\
\text { determi- } \\
\text { nados }\end{array}$ & $\begin{array}{l}\text { Locais } \\
\text { determi- } \\
\text { nados }\end{array}$ & $\begin{array}{l}\text { Em } \\
\text { qualquer } \\
\text { lugar }\end{array}$ & $\begin{array}{l}\text { Locais } \\
\text { determi- } \\
\text { nados }\end{array}$ \\
\hline $\begin{array}{l}\text { Sincronização } \\
\text { entre senso- } \\
\text { res }\end{array}$ & Não & Não & Não & Não & Não & Não & Não \\
\hline
\end{tabular}

dleware através do módulo de comunicação e armazenam os dados enviados no módulo do dispositivo. As aplicações externas acessam os dados armazenados no middleware por meio do módulo de integração, enquanto que o módulo de gerenciamento é responsável pela administração e comunicação entre os demais módulos.

O módulo de comunicação é responsável por realizar a comunicação do middleware com os sensores corporais de uma rede WBAN, conforme as configurações de energia e segurança definidas no módulo de gerenciamento. Dentro desse módulo há um componente 6 LowPAN responsável por realizar a conversão dos cabeçalhos enviados em IPv6 para o cabeçalho reduzido utilizado pelo padrão IEEE 802.15.4 e pelo padrão Bluetooth. Esse módulo também é responsável por fornecer uma comunicação energeticamente mais eficiente entre o smartphone e os sensores de uma WBAN, através da redução do ciclo de trabalho dos sensores que é oferecido pelos protocolos BLE e IEEE 802.15.4.

A comunicação por meio de ciclos de trabalho também permite a sincronização entre os sensores de uma WBAN, através do modelo de ordem de eventos utilizado no trabalho de [Djenouri and Bagaa 2016], onde os dados recebidos por cada sensor são reordenados conforme seu intervalo de chegada. Isso evita troca de dados de sincronização entre middleware e sensores, poupando energia desses dispositivos. A reordenação dos dados recebidos segue a seguinte metodologia:

- É registrado o horário de chegada (timestamp) dos dados de um determinado sensor;

- É calculado o intervalo de transmissão de cada sensor, a partir do cálculo da diferença entre os dois últimos instantes de recebimento de dados; 


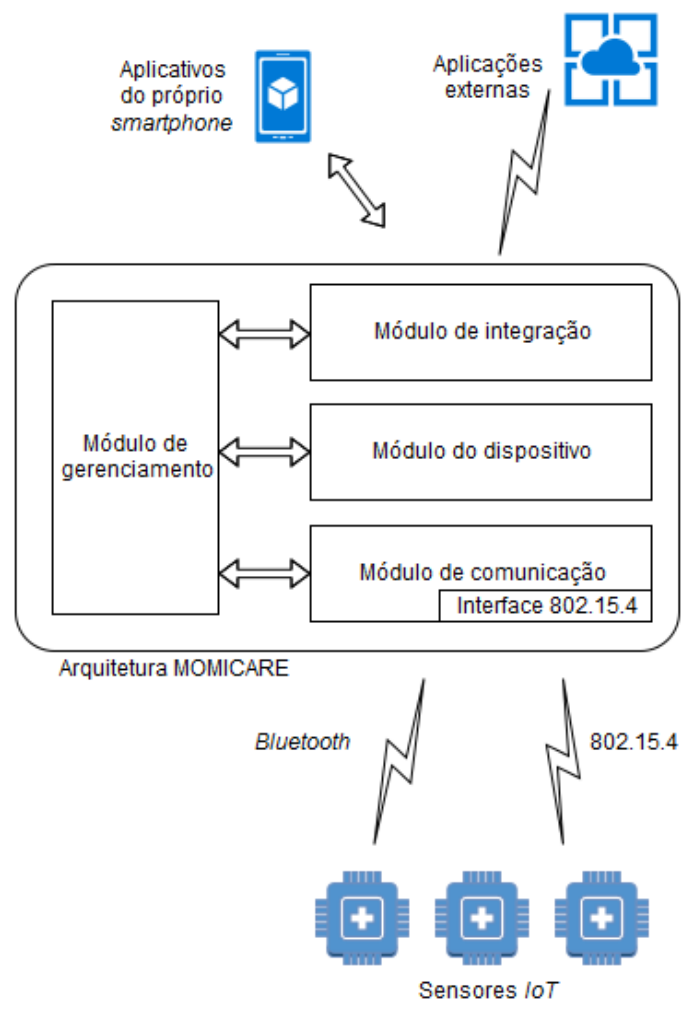

Figura 2. A arquitetura da solução MOMICARE.

- São analisados os horários recebidos na última transmissão e calculado o intervalo obtido entre o horário do primeiro e do último dado recebido do sensor nesse horário;

- É calculado a média da diferença entre o intervalo de transmissão e o intervalo dos dados;

- É registrado o horário consolidado, somando o valor obtido da média ao horário de chegada dos dados.

O módulo do dispositivo do MOMICARE é responsável por administrar os recursos do smartphone que serão utilizados pelo middleware, como os sensores instalados no aparelho, a bateria, o armazenamento interno e os hardwares de comunicação existentes. Ele atende às requisições solicitadas pelo módulo de gerenciamento e relata a este informações diversas referentes aos recursos utilizados pelo middleware, tais como carga da bateria, quantidade de espaço livre no armazenamento interno e os sensores disponíveis no smartphone. Esse módulo também é responsável por armazenar os dados coletados pelos sensores e sincronizados pelo módulo de comunicação.

O módulo de integração tem a função de realizar a comunicação entre o middleware e os aplicativos de terceiros instalados no próprio smartphone, em smartphones externos ou em qualquer outro dispositivo, a partir das configurações definidas no módulo de gerenciamento. Caso a solicitação de comunicação seja enviada a uma aplicação terceira instalada no próprio smartphone, ela será efetuada por meio de chamada de sistema do próprio sistema operacional do dispositivo. Caso contrário, ela se dará por qualquer outra aplicação que possa comunicar-se pelo padrão Representational State Transfer (REST). 
Por fim, o módulo de gerenciamento é responsável pelo funcionamento do middleware. Ele coordena a comunicação entre os demais módulos e permite a configuração do middleware pelo usuário, através de uma interface. Entre as opções de configuração possíveis, estão as seguintes:

- Configuração de uso dos sensores do smartphone: permitir que o usuário ative ou desative sensores do dispositivo, fornecidos através do módulo do dispositivo, que podem auxiliar da captura de dados;

- Segurança: utilização de mecanismos de segurança no nível de enlace oferecidos pelos protocolos para segurança nas comunicações entre smartphone e rede WBAN, a serem executadas pelo módulo de comunicação;

- Configurações de energia: configurar o intervalo de captura de dados dos sensores, bem como demais parametrizações nos protocolos Bluetooth e IEEE 802.15.4 que podem auxiliar na otimização do consumo de bateria, a serem executadas pelo módulo de comunicação.

\section{Avaliação}

A avaliação foi concebida de forma a avaliar o comportamento do middleware em relação ao consumo energético, bem como analisar a viabilidade de utilização da técnica de sincronismo proposta em aplicações reais. Ela foi efetuada a partir de simulações realizadas com o emulador Cooja, disponível no sistema operacional Contiki, voltado para aplicações IoT [Contiki 2017]. O Cooja destina-se a simular redes de sensores sem fio em diversos níveis de avaliação, possibilitando assim a realização de avaliações mais detalhadas e precisas em relação ao comportamento da rede.

Para realização da avaliação do modelo foram montados três cenários que demonstram simular o acesso de quatro aplicações distintas (de um hospital, de um profissional médico, de uma ambulância e do próprio paciente) aos sensores corporais de um paciente, conforme demonstrado na Figura 3. O primeiro, chamado de Cenário 1 e mostrado na Figura 3 (a), é composto por 3 sensores IoT, chamados de sensores-filho, em amarelo, que formam a rede $W B A N$, e por outros 4 sensores, chamados de sensores-pai, em verde. Estes últimos simulam as requisições recebidas de aplicações de terceiros, por meio de um script que executa o comando ping para cada um dos sensores-filho. Dessa forma, cada um dos sensores-pai executa uma requisição por segundo a cada um dos sensores-filho. Tanto os sensores-pai quanto os sensores-filho utilizaram o sensor Tmote-sky disponível no emulador.

O segundo cenário, chamado de Cenário 2 e mostrado na Figura 3 (b), é formado pelos mesmos 3 sensores-filho que formam a rede $W B A N$ e por 2 sensores-pai. Cada um dos sensores-pai executa 2 scripts que simulam as requisições recebidas de aplicações de terceiros. Por fim, o terceiro cenário, chamado de Cenário 3 e mostrado na Figura 3 (c), é formado pelos mesmos 3 sensores-filho que formam a rede WBAN e por apenas 1 sensor-pai. Este, por sua vez, executa os quatro scripts, simulando o comportamento do MOMICARE.

Como o Cooja não possui um módulo para verificação do consumo de energia dos sensores, foi desenvolvida uma aplicação que calcula o consumo energético da interface de rádio de cada um dos sensores-filho, a partir da seguinte fórmula:

$$
\text { ConsumoEnergetico }=(T X+R X) * 20 * 3
$$




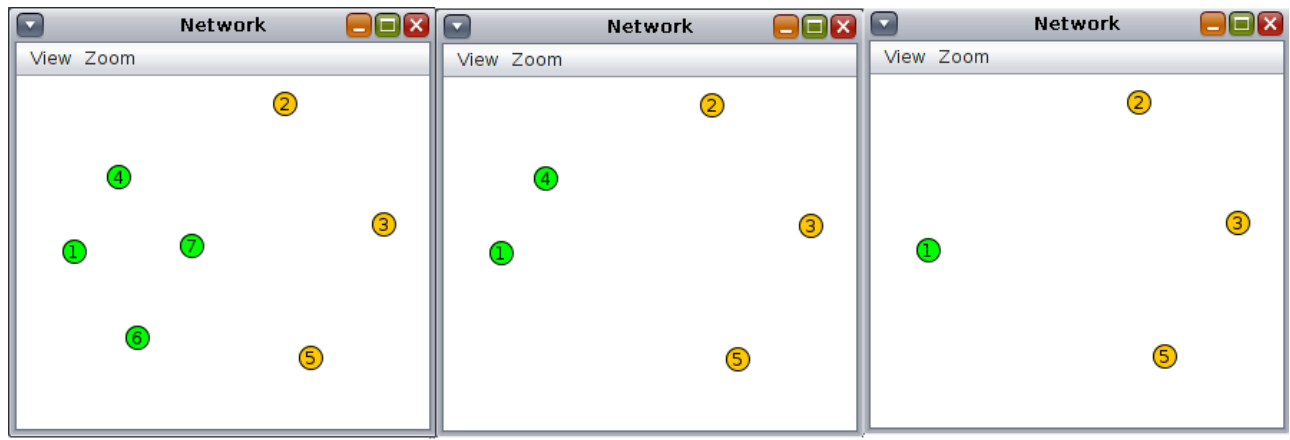

(a)

(b)

(c)

Figura 3. Os cenários de simulação. Em (a), considerando quatro aplicações independentes. Em (b), considerando duas aplicações. Em (c), considerando uma aplicação (modelo MOMICARE)

onde TX representa a porcentagem de tempo que o sensor esteve transmitindo dados; RX representa a porcentagem de tempo que o sensor esteve recebendo dados, a constante 20 representa a corrente, em miliamperes, que a interface de comunicação do sensor utiliza ao transmitir ou receber dados; e a constante 3 representa a tensão, em volts, utilizada pelo sensor. O consumo energético é dado em watts. Neste cálculo não foi considerado o consumo energético do processador de cada um dos sensores pois o objetivo desta avaliação é verificar o impacto energético a partir da comunicação entre sensores, analisada a partir dos cenários descritos nesta Seção.

Cada um desses cenários foi executado durante 1 hora no emulador. O consumo energético registrado por cada um dos sensores-filho é demonstrado na Figura 4.

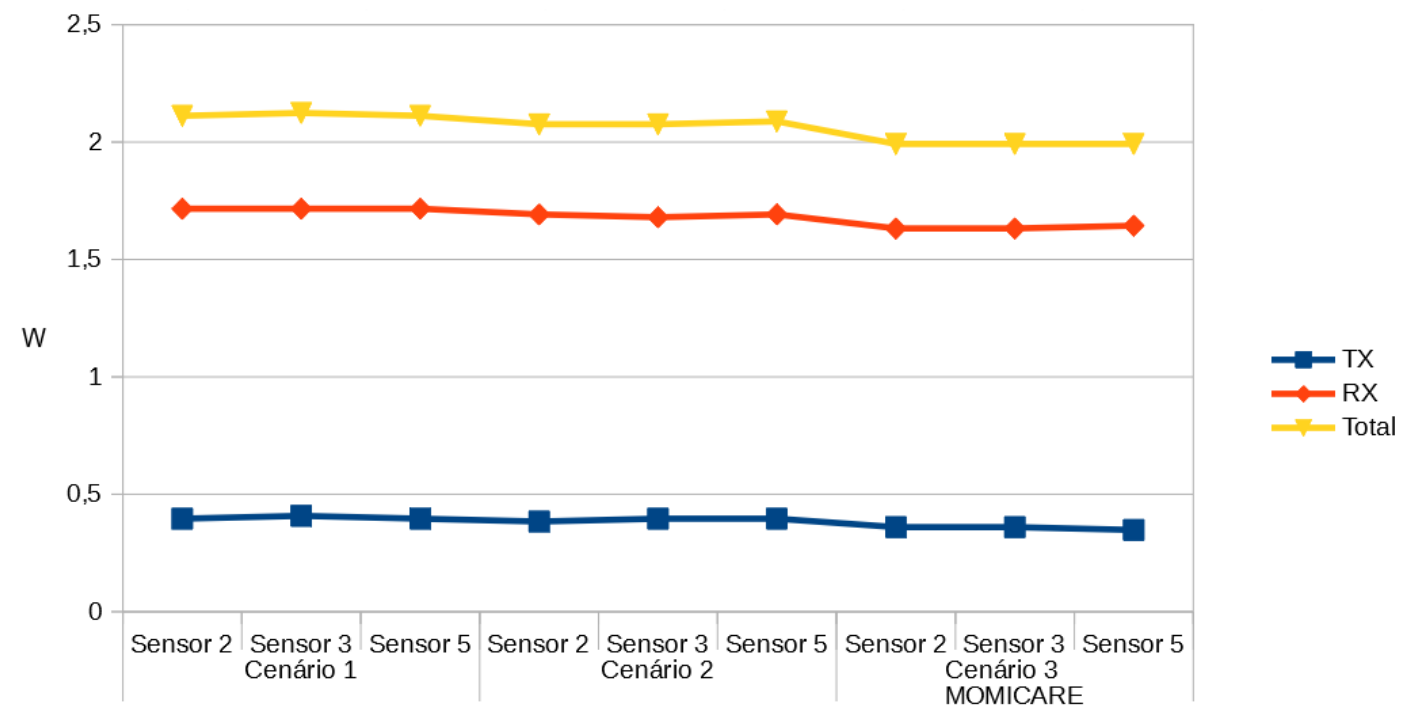

Figura 4. Consumo energético dos sensores nas simulações realizadas.

Como resultados obtidos, verificou-se primeiramente que a simulação considerando o cenário de uso do MOMICARE apresentou uma redução de 4,2\% no consumo energético dos sensores-filho em relação ao Cenário 2 e de 5,9\% em relação ao Cenário 1. Isso demonstra que somente pelo uso de um canal único de comunicação entre os sensores e as aplicações já apresenta benefícios em relação ao consumo de energia. 
Além disso, verificou-se também a possibilidade de implementar a sincronização por ordem de eventos de acordo com a metodologia descrita no modelo. Conforme exposto na Figura 5, há o registro do horário (timestamp) em que houve trocas de dados entre os sensores-pai e filho, bem como a informação entre quais sensores essa troca ocorreu.

A análise efetuada a partir dessa simulação também permitiu verificar que o middleware pode tornar-se escalável replicando-se os dados entre vários smartphones, conforme já demonstrado no trabalho de [Castillejo et al. 2013]. Por fim, a segurança na comunicação pode ser implementada através dos recursos contidos nos protocolos de enlace [IEEE 2011] [SIG 2016].

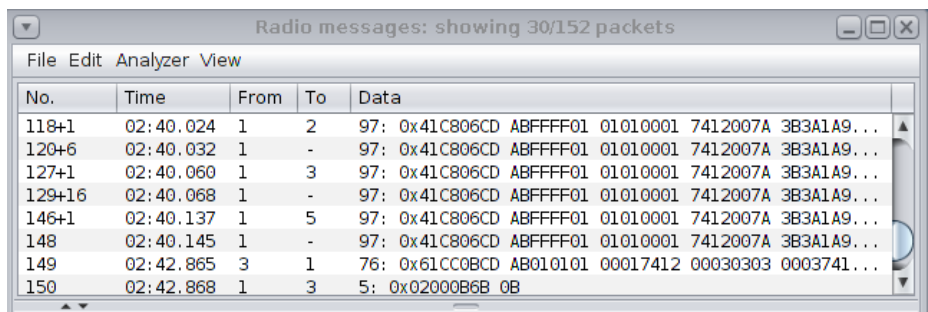

Figura 5. Registros dos horários em que houve troca de dados entre os sensores.

\section{Conclusão}

O uso de sensores corporais para cuidados médicos apresenta diversos desafios, como o sincronismo entre os sensores e a duração da bateria desses dispositivos. Um resposta a isso é apresentado no modelo MOMICARE, um middleware instalado num dispositivo móvel - como um smartphone - que recebe os dados de diversos sensores corporais, sincroniza-os e armazena-os, retransmitindo-os às aplicações médicas que solicitem esses dados. Nos cenários avaliados, verificou-se que o MOMICARE reduziu o consumo de energia dos sensores IoT corporais, bem como constatou-se a possibilidade de realizar o sincronismo entre os dados recebidos pelos diversos sensores a partir da metodologia apresentada no modelo.

Como trabalhos futuros, pretende-se desenvolver um protótipo que valide os dados preliminares obtidos a partir dessa simulação, bem como avaliar se as lacunas de segurança e escalabilidade dos sensores também podem ser suportados pelo middleware sem prejuízos no que diz respeito ao consumo energético dos sensores e à sincronização entre esses dispositivos.

\section{Agradecimentos}

Os autores gostariam de agradecer ao CNPq pelo apoio a esta pesquisa.

\section{Referências}

Castillejo, P., Martinez, J. F., Rodriguez-Molina, J., and Cuerva, A. (2013). Integration of wearable devices in a wireless sensor network for an E-health application. IEEE Wireless Communications, 20(4):38-49.

Contiki (2017). Contiki open source operating system. Disponível em: $<$ http://www.contiki-os.org >. Acesso em: 05.fev.2017. 
Djenouri, D. and Bagaa, M. (2016). Synchronization protocols and implementation issues in wireless sensor networks: A review. IEEE Systems Journal, 10(2):617-627.

Goswami, S., Roy, P., Dey, N., and Chakraborty, S. (2016). Wireless body area networks combined with mobile cloud computing in healthcare: A survey. In Classification and Clustering in Biomedical Signal Processing, pages 388-402. IGI Global.

IBGE, I. B. d. G. e. E. (2015). Séries estatísticas e históricas. Disponível em: $<$ http://seriesestatisticas.ibge.gov.br/series.aspx?no=13\&op=0\&vcodigo=MS44 $>$. Acesso em: 29.set.2015.

IEEE (2011). 802.15.4 Low-Rate Wireless Personal Area Networks (LR-WPANs), volume 2011.

Jung, E. Y., Kim, J. H., Chung, K. Y., and Park, D. K. (2013). Home health gateway based healthcare services through U-health platform. Wireless Personal Communications, 73(2):207-218.

Lane, N. D., Lin, M., Mohammod, M., Yang, X., Lu, H., Cardone, G., Ali, S., Doryab, A., Berke, E., Campbell, A. T., et al. (2014). Bewell: Sensing sleep, physical activities and social interactions to promote wellbeing. Mobile Networks and Applications, 19(3):345-359.

Lee, Y.-G., Jeong, W. S., and Yoon, G. (2012). Smartphone-Based Mobile Health Monitoring. Telemedicine and e-Health, 18(8):585-590.

Maia, P., Baffa, A., Cavalcante, E., Delicato, F. C., Batista, T., and Pires, P. F. (2015). A middleware platform for integrating devices and developing applications in e-health. In Computer Networks and Distributed Systems (SBRC), 2015 XXXIII Brazilian Symposium on, pages 10-18. IEEE.

Merrell, R. C. and Doarn, C. R. (2014). m-Health. Telemedicine and e-Health, 20(2):99101.

Moser, L. E. and Melliar-Smith, P. (2015). Personal health monitoring using a smartphone. In 2015 IEEE International Conference on Mobile Services, pages 344 351. IEEE.

Riazul Islam, S. M., Humaun Kabir, M., and Hossain, M. (2015). The internet of things for health care: A comprehensive survey. IEEE Access, 3:678-708.

Seeger, C., Van Laerhoven, K., and Buchmann, A. (2015). Myhealthassistant: An eventdriven middleware for multiple medical applications on a smartphone-mediated body sensor network. IEEE journal of biomedical and health informatics, 19(2):752-760.

Shankar, S. K. and Tomar, A. S. (2016). A survey on wireless body area network and electronic-healthcare. In Recent Trends in Electronics, Information \& Communication Technology (RTEICT), IEEE International Conference on, pages 598-603. IEEE.

SIG, B. S. I. G. (2016). Bluetooth low energy. Disponível em: $<$ https://www.bluetooth.com/what-is-bluetooth-technology/bluetooth-technologybasics/low-energy $>$. Acesso em: 15.set.2016.

Wang, K. I. K., Dubey, S., Rajamohan, A., and Salcic, Z. (2015). An Android-Based Mobile 6LoWPAN Network Architecture for Pervasive Healthcare. Proceedings - 2015 International Conference on Intelligent Environments, IE 2015, pages 49-56. 